\title{
Assessment of Spontaneous Cardiovascular Oscillations in Parkinson's Disease
}

\author{
Gaetano Valenza \#, Stefano Orsolini, Stefano Diciotti, Luca Citi, Enzo Pasquale Scilingo, Maria \\ Guerrisi, Sabrina Danti, Claudio Lucetti, Carlo Tessa, Riccardo Barbieri*, Nicola Toschi* \\ * These authors contributed equally to this work. \\ \# Corresponding author: Gaetano Valenza. Email: g.valenza@ieee.org
}

G. Valenza, R. Barbieri, and N. Toschi are with the Massachusetts General Hospital-Harvard Medical School, Boston, USA.

L. Citi is with the School of Computer Science and Electronic Engineering, University of Essex, Colchester, UK.

G. Valenza and E.P. Scilingo are with the Department of Information Engineering, and Research Centre "E. Piaggio", University of Pisa, Pisa, Italy.

M. Guerrisi and N. Toschi are with the Department of Biomedicine and Prevention, University of Rome "Tor Vergata", Rome, Italy.

S. Orsolini and S. Diciotti are with the Department of Electrical, Electronic, and Information Engineering "Guglielmo Marconi", University of Bologna, Cesena, Italy

S. Danti, C. Lucetti and C. Tessa are with the Versilia Hospital, Viareggio, Italy.

R. Barbieri is with the Department of Electronics, Informatics and Bioengineering, Politecnico di Milano, Milano. Italy.

Key Words: Parkinson's disease, Heart Rate Variability, Autonomic Nervous System, Point process, Laguerre expansion, Bispectrum, Lyapunov Exponents, Support Vector Machine, Autonomic dysfunction 


\section{Abstract}

Parkinson's disease (PD) has been reported to involve postganglionic sympathetic failure and a wide spectrum of autonomic dysfunctions including cadiovascular, sexual, bladder, gastrointestinal and sudo-motor abnormalities. While these symptoms may have a significant impact on daily activities, as well as quality of life, the evaluation of autonomic nervous system (ANS) dysfunctions relies on a large and expensive battery of autonomic tests only accessible in highly specialized laboratories. In this paper we aim to devise a comprehensive computational assessment of disease-related heartbeat dynamics based on instantaneous, time-varying estimates of spontaneous (resting state) cardiovascular oscillations in PD. To this end, we combine standard ANS-related heart rate variability (HRV) metrics with measures of instantaneous complexity (dominant Lyapunov exponent and entropy) and higher-order statistics (bispectra). Such measures are computed over 600-s recordings acquired at rest in 29 healthy subjects and 30 PD patients. The only significant group-wise differences were found in the variability of the dominant Lyapunov exponent. Also, the best PD vs. healthy controls classification performance (balanced accuracy: $73.47 \%$ ) was achieved only when retaining the time-varying, non-stationary structure of the dynamical features, whereas classification performance dropped significantly (balanced accuracy: 61.91\%) when excluding variability-related features. Additionally, both linear and nonlinear model features correlated with both clinical and neuropsychological assessments of the considered patient population. Our results demonstrate the added value and potential of instantaneous measures of heartbeat dynamics and its variability in characterizing PD-related disabilities in motor and cognitive domains. 


\section{Introduction}

Parkinson's disease (PD) is the second most common neurodegenerative disorder after Alzheimer's disease, and is classically associated with motor symptoms including tremor, balance problems, limb rigidity, bradykinesia and gait abnormalities [12]. The causes and aetiology of PD are still largely unknown, and current literature is inconclusive when it comes to isolating reliable predictors of disease incidence and evolution. In this context, older age (> 60 years), iposmia, REM sleep behavior disorder, constipation, and depression are the major risk factors for PD that have been consistently found across studies [12] [43].

PD is frequently associated with symptoms of Autonomic Nervous System (ANS) dysfunction and/or failure [26], including cardiovascular, sexual, bladder, gastrointestinal, and sudo-motor abnormalities [2]. These clinical evidences are in agreement with the results of pathological studies demonstrating how the degenerative process in PD involves subcortical structures, brainstem and medullary autonomic centres as well as post-ganglionic sympathetic fibers [30]. Accordingly, Lewy bodies (the pathological hallmark of PD) have been found in a number of ANS regions such as the hypothalamus and the sympathetic/parasympathetic systems $[44,45]$.

While cardiovascular dysautonomia has traditionally been associated with the later stages of PD, it has recently become evident that it can occur at any disease stage, and that its onset can even precede the appearance of motor symptoms $[2,18,50]$. Previous studies have reported a variable prevalence of cardiovascular autonomic dysfunction in PD, ranging from $23 \%$ to $80 \%$ as determined by studies which included cohorts of between 20 and 91 patients [3, $29,35]$. Within these reports, conflicting evidence exists about the extent and characteristics of PD-related aberrations in cardiovascular control. As an example, two studies have described orthostatic hypotension and a decreased heart rate response to the Valsalva maneuver $[1,28]$, while other authors have found that pressure and heart rate response during these procedures remained normal $[25,33]$.

It is well know that ANS outflow can be estimated non-invasively using Heart Rate Variability (HRV) analysis [42], and recently HRV measures have been employed to explore ANS alterations in PD by evaluating the modulatory effects of ANS dynamics on sinus node activity 
[21]. According to the study by Haapaniemi et al. [16], when analyzing $24 \mathrm{~h}$ ECG recordings all HRV spectral components were found to be significantly lower in PD patients when compared to controls [16]. In another study, which evaluated 10 consecutive minutes of resting ECG recordings, HRV High Frequency (HF) power (thought to mainly reflect parasympathetic activity) was significantly different between healthy controls and untreated (i.e., de novo) PD patients, whereas nonlinear HRV analysis based on entropy and geometrical measures did not show any statistical differences between the two groups [20]. However, other authors documented an increase in complexity of systolic arterial pressure in PD patients when compared to controls [32]. In addition, while the physiology underlying complexity-related measures of heartbeat dynamics is still unknown, it is well known that their quantification can provide meaningful information on psychophysiological as well as pathological states [8, 10, 14, 24, 39]. In this context, we have previously shown that measures of time-varying complexity provide enhanced discriminatory power when compared to standard complexity measures [36, 38], and that the stability and complexity of autonomic dynamics is altered in PD patients when compared to healthy controls as well as within different PD subgroups $[4,6]$.

In this paper we aim to devise a comprehensive assessment of disease-related ANS alterations based on instantaneous, time-varying estimates of spontaneous (resting state) cardiovascular oscillations in PD. To this end, we employ our instantaneous inhomogeneous point-process framework [36-38], which augments standard ANS-related HRV metrics defined in the time and frequency domains [5] with measures of instantaneous complexity (dominant Lyapunov exponent and entropy) [36, 38] and higher-order statistics (bispectra) [37]. Additionally, in order to develop a processing chain in line with the clinical need of single-subject classification, we complement the study of group-wise statistical differences with the training and validation of an automatic classification algorithm which includes a feature selection procedure.

The rest of this paper is organized as follows: in section 2 we provide details about the methods employed for data acquisitions, patient assessment, probabilistic modelling of the heartbeat, extraction of linear and nonlinear features and classification analysis. In section 3 , 
we illustrate the results of these procedures in the same order, and in section 4 we discuss the implication of our results for characterising cardiovascular dysautonomia in PD through non-invasive, instantaneous HRV modeling. 


\section{Materials and methods}

\subsection{Experimental Setup}

Plethysmographic (PPG) signals were recorded with a sample frequency of $50 \mathrm{~Hz}$ from 29 healthy controls (HC, 18 males, 11 females, age $60.63 \pm 4.93$ years, median \pm MAD, where $\operatorname{MAD}(\mathrm{X})=\operatorname{Median}(|\mathrm{X}-\operatorname{Median}(\mathrm{X})|))$ and 30 PD patients (23 males, 7 females, age $66.51 \pm$ 4.06 years, median \pm MAD). Between the two groups, no significant difference was found in age ( $p=0.15$, Mann-Whitney test, null-hypothesis of equal medians) and gender $(p=0.46$, Chi Square test, null-hypothesis of no gender effect). Subjects were placed horizontally in a supine position and remained at rest during the whole recording (600s). During the acquisition, all subjects were instructed not to talk and maintained relaxed spontaneous breathing. All participants gave written informed consent to participating in the study, which was approved by the local ethics committee. An overview of the experimental set-up and block scheme of the overall signal processing and classification chain is shown in Fig. 1.

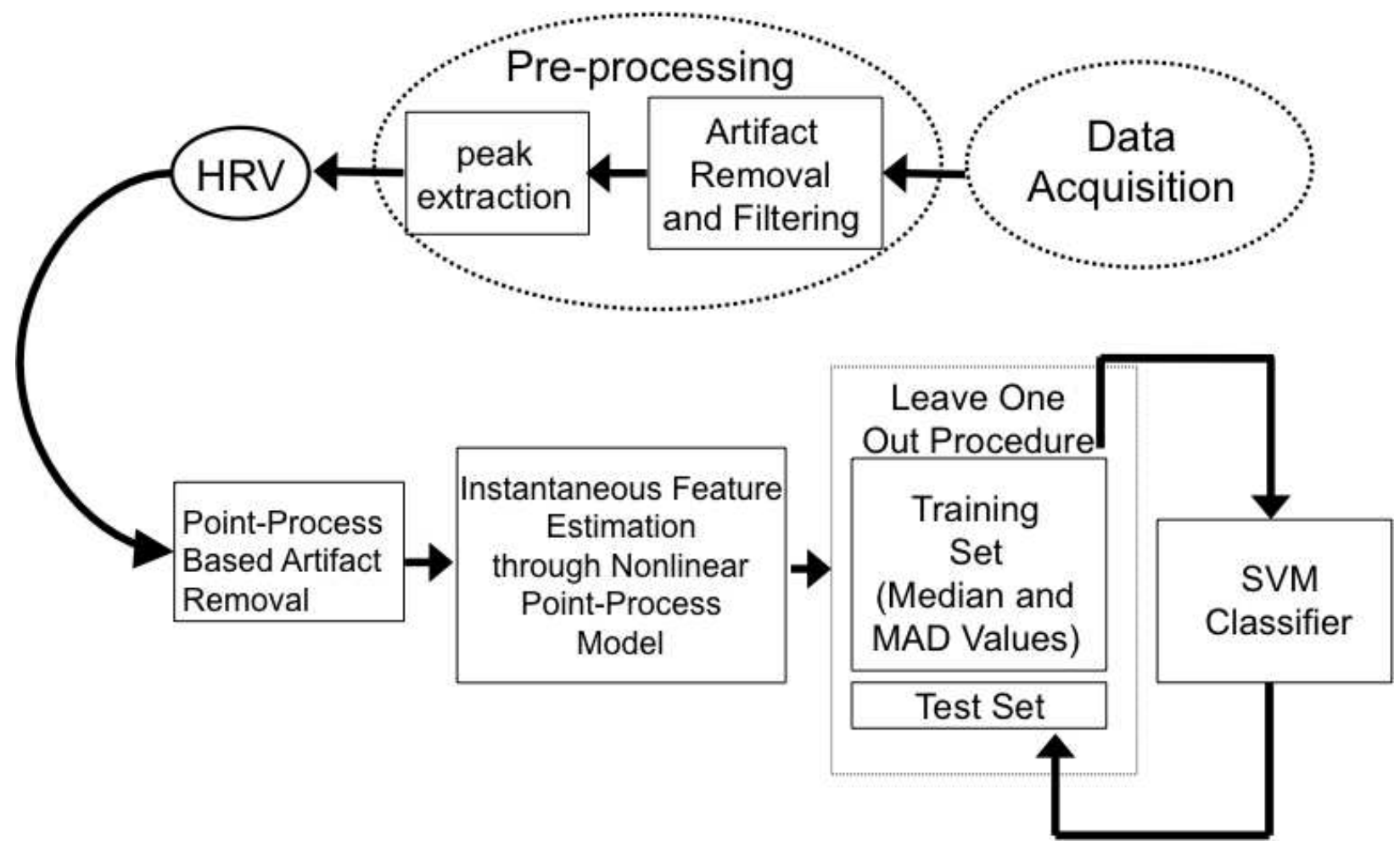

Figure 1: Overview of the experimental set-up and block scheme of the overall signal processing and classification chain. Starting from data acquisition, HRV series are extracted by using automatic peak detection algorithms 
applied on artifact-free signals. The absence of both algorithmic errors (e.g., mis-detected peaks) or ectopic beats is ensured by the application of effective artifact removal methods as well as visual inspection. The nonlinear point-process model is fitted to the HRV series, and all features are estimated in an instantaneous fashion. Successively, for each subject, a feature set is defined and fed into support vector machine-based classification using leave-one our procedures.

\subsection{Patient Assessment}

Clinical assessment included history of disease's related symptoms and signs, and full neurological examination. All patients were screened for cardiovascular autonomic dysfunction which was considered as exclusion criterion. All patients had to satisfy the UK Brain Bank criteria for the diagnosis of PD [13] and were in stage 1, 1.52 or 2.5 according to the Hoehn\&Yahr (HY) system. As supportive criterion, a 123IFP-CIT SPECT to confirm nigrostriatal degeneration was performed. With the aim of exploring how much information about clinical disability can be extracted from our HRV assesment framework, severity of parkinsonism was evaluated by the Unified Parkinson's Disease Rating Scale (UPDRS) [31] and the HY staging system [17]. Possible abnormalities in the affective domain were investigated through the Geriatric Depression Scale (GDS). Patient population was comprised of 15 de novo PD patients (i.e. naive/drug free patients in the initial stage of the disease) and 15 patients with more advanced PD, which necessarily were on dopaminergic drugs (i.e. dopamine agonist and levodopa therapy, no other drugs were permitted). No patients of these 15 treated PD showed complications such as wearing-off, on-off and/or dyskinesia. Moreover, in order to avoid the possible interference of levodopa on cardiac function, we have assessed HRV measures in the morning before the first levodopa dose. Only one patient had a HY score $>=2$, whereas all other patients had 1 or 1.5 . The overall disease duration was 1.6 years (standard deviation in 0.35 years).

Additionally, in order to explore putative associations between our HRV-related features and PD-related disabilities in the cognitive domain, we evaluated our patients through a pool of standardized neuropsychological tests that included at least two tests within each of the five cognitive domains [51]. Specifically, we examined: 1) attention and working memory domain (by Trail Making Test, $T M T_{-} B$, and Stroop Test, Stroop_E, - both errors and time parameters), 2) executive functions (by Frontal Assessment Battery, FAB, Wechsler Adult Intelligence Scale 
Analogy Subtest, Analogie_W, and Brixton Spatial Anticipation Test), 3) language (by Boston Naming Test, Boston_60, and Phonemic Fluency), 4) memory (by Memory Prose and Verbal Learning Pair Task), 5) visuospatial ability (by Wechsler Adult Intelligence Scale Drawing Cubes Subtest and Benton Judgment of Line Orientation, Benton_JLO). Global cognitive status was assessed with the Montreal Cognitive Assessment.

\subsection{Point-Process Model of the Heartbeat}

By using the inhomogeneous point process model of heartbeat dynamics [5], we model the unevenly sampled RR interval series through probability density functions ( $p d f$ ) characterizing and predicting the time until the next event occurs as a function of the past history. Within this framework, Laguerre expansions of the Wiener-Volterra linear and nonlinear autoregressive terms account for long-term nonlinear information [36-38]. As major advantages, instantaneous measures can be estimated without applying any interpolation techniques to the RR interval series, and are associated to effective goodness-of-fit measures.

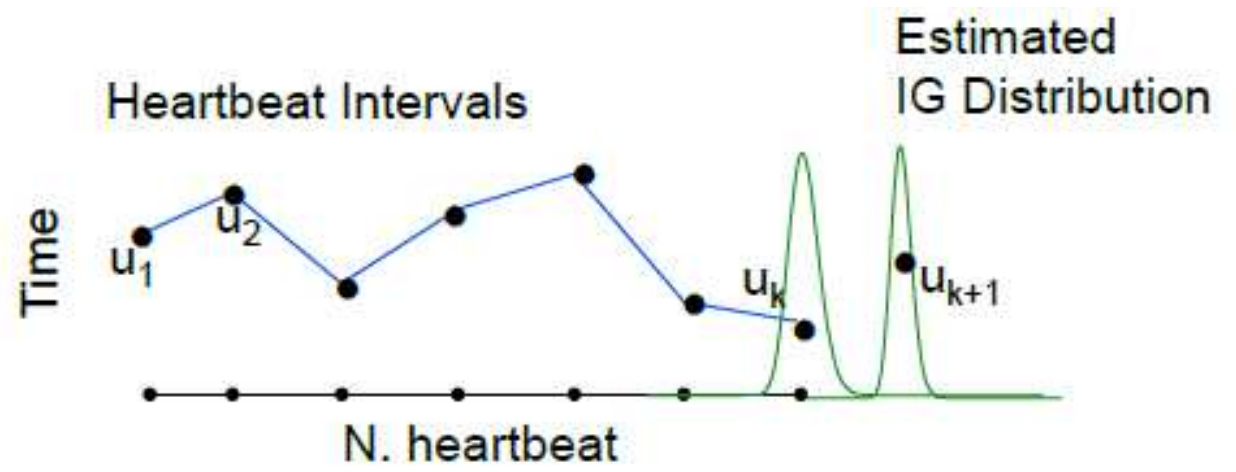

Figure 2: A graphical representation of point-process modeling of heartbeat dynamics. The horizontal axis represents the counting process along the number of heartbeats, whereas the vertical axis represents the duration of heartbeat intervals. Inverse-Gaussian (IG) distributions (green lines on the right) characterize the prediction of the future heartbeat event along the time.

In general, a random point process is a stochastic process comprising the occurrence in time of discrete events, which in our case are represented by the heartbeats. As a consequence, it is possible to characterize the $p d f$ of the next ventricular contraction through a parametric formulation of the past heartbeat events (see Fig. 2). Motivated by both 
goodness-of-fit as well as by physiological reasons, an inverse Gaussian pdf was used to model the R-beat series [5]:

$$
f\left(t \mid \mathscr{H}_{t}, \xi(t)\right)=\sqrt{\frac{\xi_{0}(t)}{2 \pi\left(t-u_{j}\right)^{3}}} \exp \left\{-\frac{1}{2} \frac{\xi_{0}(t)\left[t-u_{j}-\mu_{\mathrm{RR}}\left(t, \mathcal{H}_{t}, \xi(t)\right)\right]^{2}}{\mu_{\mathrm{RR}}\left(t, \mathcal{H}_{t}, \xi(t)\right)^{2}\left(t-u_{j}\right)}\right\}
$$

where:

- $t \in(0, T]$, the observation interval;

- $0 \leq u_{1}<\cdots<u_{k}<u_{k+1}<\cdots<u_{K} \leq T$ the times of the events;

- $\mathrm{RR}_{j}=u_{j} u_{j-1}>0$ the $j^{\text {th }} \mathrm{RR}$ interval;

- $\left\{u_{j}\right\}_{j=1}^{J}$ the R-wave events, and $j=\widetilde{N}(t)$ the index of the previous R-wave event before time $t$

- $N(t)=\max \left\{k: u_{k} \leq t\right\}$ the sample path of the counting process of the RR interval series;

- $\widetilde{N}(t)=N\left(t^{-}\right)=\lim _{\tau \rightarrow t^{-}} N(\tau)=\max \left\{k: u_{k}<t\right\}$

- $\mathcal{H}_{t}=\left(u_{j}, \mathrm{RR}_{j}, \mathrm{RR}_{j-1}, \ldots, \mathrm{RR}_{j-M+1}\right)$;

- $\xi(t)$ the vector of the model time-varying parameters;

- $\mu_{\mathrm{RR}}\left(t, \mathscr{H}_{t}, \xi(t)\right)$ the first-moment statistic (mean) of the distribution;

- $\xi_{0}(t)>0$ the shape parameter of the inverse Gaussian distribution;

Accounting for history dependence, $f\left(t \mid \mathscr{H}_{t}, \xi(t)\right)$ is thus able to predict the next heartbeat event. This function is parametrized in its first-order moment as a Nonlinear Autoregressive model with Laguerre expansions (NARL) of the Volterra terms:

$$
\begin{gathered}
\mu_{\mathrm{RR}}\left(t, \mathcal{H}_{t}, \xi(t)\right)=\mathrm{RR}_{\widetilde{N}(t)}+g_{0}(t)+\sum_{i=0}^{p} g_{1}(i, t) l_{i}\left(t^{-}\right)+ \\
\sum_{i=0}^{q} \sum_{j=0}^{q} g_{2}(i, j, t) l_{i}\left(t^{-}\right) l_{j}\left(t^{-}\right) .
\end{gathered}
$$

where

$$
l_{i}\left(t^{-}\right)=\sum_{n=1}^{\widetilde{N}(t)} \phi_{i}(n)\left(\operatorname{RR}_{\widetilde{N}(t)-n}-\operatorname{RR}_{\widetilde{N}(t)-n-1}\right)
$$

is the output of the Laguerre filters just before time $t$, and

$$
\phi_{i}(n)=\alpha^{\frac{n-i}{2}}(1-\alpha)^{\frac{1}{2}} \sum_{k=0}^{i}(-1)^{k}\left(\begin{array}{l}
n \\
k
\end{array}\right)\left(\begin{array}{l}
i \\
k
\end{array}\right) \alpha^{i-k}(1-\alpha)^{k},(n \geq 0)
$$

is the $i^{\text {th }}$-order discrete time orthonormal Laguerre function and $\alpha$ is the discrete-time Laguerre parameter $(0<\alpha<1)$ which determines the rate of exponential asymptotic decline.

The coefficients $g_{0},\left\{g_{1}(i)\right\}$ with $\mathrm{i}=0, \ldots \mathrm{p}$, and $\left\{g_{2}(i, j)\right\}$ with $\{\mathrm{i}, \mathrm{j}\}=0, \ldots, \mathrm{q}$ correspond to the time-varying zero-, first-, second-order NARL coefficients, respectively, and performing the Laguerre expansion on the derivative R-R series improves stationarity within the 
sliding time window $W$ (in this work we have chosen $W=90$ s) $[15,37]$. The corresponding nonlinear autoregressive Volterra-Wiener long-term memory model with second-order nonlinearity becomes [27]:

$$
\begin{aligned}
& \mu_{\mathrm{RR}}\left(t, \mathcal{H}_{t}, \xi(t)\right)= \mathrm{RR}_{\widetilde{N}(t)}+\gamma_{0} \\
& \quad+\sum_{i=1}^{\infty} \gamma_{1}(i, t)\left(\mathrm{RR}_{\widetilde{N}(t)-i}-\mathrm{RR}_{\widetilde{N}(t)-i-1}\right) \\
&+\sum_{i=1}^{\infty} \sum_{j=1}^{\infty} \gamma_{2}(i, j, t)\left(\mathrm{RR}_{\widetilde{N}(t)-i}-\mathrm{RR}_{\widetilde{N}(t)-i-1}\right) \\
& \times\left(\operatorname{RR}_{\widetilde{N}(t)-j}-\mathrm{RR}_{\widetilde{N}(t)-j-1}\right)
\end{aligned}
$$

As $\mu_{\mathrm{RR}}\left(t, \mathscr{H}_{t}, \xi(t)\right)$ is defined in continuous time, it is possible to obtain an instantaneous $\mathrm{R}-\mathrm{R}$ mean estimate at arbitraty timescales without interpolating between the arrival times of two consecutive heartbeats.

\subsubsection{Parameter Estimation, Model Selection, Goodness-of-Fit}

Given a local observation interval $(t-l, t]$ of duration $l$, we consider a subset $U_{m: n}$ of the R-wave events, where $m=N(t-l)+1$ and $n=N(t)$ and, at each time $t$, we find the unknown time-varying parameter vector $\xi(t)$ that maximizes the local log-likelihood [5]:

$$
\begin{aligned}
& L\left(\xi(t) \mid U_{m: n}\right)=\sum_{k=m+p-1}^{n-1} w\left(t-u_{k+1}\right) \\
& \quad \log \left[f\left(u_{k+1} \mid \mathcal{H}_{u_{k+1}}, \xi(t)\right)\right]+\log \int_{t}^{\infty} f\left(\tau \mid \mathcal{H}_{t}, \xi(t)\right) d \tau
\end{aligned}
$$

where $w(\tau)=e^{-\varpi \tau}$ is an exponential weighting function for the local likelihood. The constant $\varpi$ (together with $l$ ) governs the degree of influence of previous observations on the local likelihood and determines the trade-off between the accuracy of the estimation of the regression parameters (small $\varpi$ ) and the responsiveness to non-stationarities (large $\varpi)$. In Eq. 6 , the first summation term accounts for the past, completely-observed, R-R intervals information, while the latter term accounts for the next, not yet observed, R-R interval (right censoring). We use a Newton-Raphson procedure to maximize the local log-likelihood in Eq. 6, and compute the local maximum-likelihood estimate of $\xi(t)$ [5]. Because there is significant overlap between adjacent local likelihood intervals, we initialize the Newton-Raphson procedure at $t$ with the previous local maximum-likelihood estimate at time $t-\Delta$, where $\Delta$ defines the time interval shift to compute the next parameter update. We determine the 
optimal order $\{p, q\}$ based on the Akaike Information Criterion (AIC) and by prefitting the point process model goodness-of-fit to a subset of the data [5] and evaluating the Kolmogorov-Smirnov (KS) test and associated KS statistics [5]. At each iteration, the recursive, causal nature of the estimation allows to independently predict each new observation, given the previous history. The model and all its parameters are therefore also updated without priors at each iteration. In other words, each test point $\mathrm{RR}_{k}$ is tested against one instance of a time-varying model trained with points $\left\{\mathrm{RR}_{j}\right\}$ with $j<k$.

Autocorrelation plots are also visually inspected to ensure that all points of the plot are within the $95 \%$ of the confidence interval, hence guaranteeing the independence of the model-transformed intervals [5]. In order to perform model order selection, we integrated the KS and autocorrelation analysis [5] by evaluating the AIC criterion using the whole recordings [5]. Specifically, model orders were chosen to minimize KS distance. When more than one order was associated to the same KS distance values, optimal model orders were chosed to minimize the AIC criterion. Once the order $\{p, q\}$ is determined, the initial NARL coefficients are estimated by the method of least squares [40].

\subsubsection{Heartbeat Correction}

In order to provide reliable results, the HRV processing techniques require uninterrupted series of RR intervals. Nevertheless, peak detection errors and ectopic beats often determine abrupt changes in the $R-R$ interval series that may result in substantial deviations of the model-derived HRV indices and potentially bias statistical outcomes from intra- and inter-subject comparisons. To eliminate such anomalies we preprocessed all heartbeat data with a previously developed real-time R-R interval error detection and correction algorithm [9] based on the point process statistics (local likelihood). Briefly, the algorithm determines whether the actual observation is in agreement with the resulting model or if, instead, the alternative hypothesis of an erroneous beat is more likely.

\subsection{Instantaneous Time, Frequency, and Higher-Order Spectral Analysis}

In order to provide quantitative tools related to standard measures defined in the time 
and frequency domains, higher order spectral representations, and complexity, it is necessary to link the NARL model to the traditional input-output Wiener-Volterra model [37]. Just like for any linear autoregressive model one can define equivalent infinite-memory moving average model, a quadratic NARL model can be linked to an input-output Volterra model, driven by the same NARL noise term. Then, the time-domain characterization is based on the first and the second order moments of the underlying probability structure. Given the time-varying parameter set $\xi(t)$, the instantaneous estimates of mean $\mu_{R R}$, and $\mathrm{R}-\mathrm{R}$ interval standard deviation $\sigma_{R R}$ can be extracted at each moment in time [5].

Estimates in the frequency domain reveal the linear mechanisms governing heartbeat dynamics in the frequency domain. In particular, given the input-output Volterra kernels of the NARL model for the instantaneous R-R interval mean $\mu_{\mathrm{RR}}\left(t, \mathcal{H}_{t}, \xi(t)\right)$, we can compute the time-varying parametric (linear) autospectrum $\mathcal{Q}(f, t)$ [37]. By integrating $\mathcal{Q}(f, t)$ in each frequency band, we can compute the power within the very low frequency (VLF = 0.01-0.05 $\mathrm{Hz})$, low frequency $(\mathrm{LF}=0.05-0.15 \mathrm{~Hz})$, and high frequency $(\mathrm{HF}=0.15-0.5 \mathrm{~Hz}$ ) ranges, along with their ratio (LF/HF). It has been well-recognized that the HF power is a reliable marker of parasympathetic activity [42]. Conversely, the LF power might be affected by both sympathetic and parasympathetic activities [42].

The higher-order spectral representation allows for the estimation of statistics beyond the second order, and phase relations between frequency components which would otherwise be suppressed. A detailed description of the instantaneous bispectrum $\left(B\left(f_{1}, f_{2}, t\right)\right)$ derivation from point-process nonlinear models can be found in [37]. This tool allows us to evaluate the instantaneous presence of nonlinearity in heartbeat series by calculating nonlinear sympatho-vagal interactions. Specifically, by integrating $\left|B\left(f_{1}, f_{2}, \mathrm{t}\right)\right|$ in the appropriate frequency bands, it is possible to obtain:

$$
\begin{array}{r}
\mathrm{LL}(t)=\int_{f_{1}=0^{+}}^{0.15} \int_{f_{2}=0^{+}}^{0.15} \mathrm{~B}\left(f_{1}, f_{2}, t\right) d f_{1} d f_{2} \\
\mathrm{LH}(t)=\int_{f_{1}=0^{+}}^{0.15} \int_{f_{2}=0.15^{+}}^{0.4} \mathrm{~B}\left(f_{1}, f_{2}, t\right) d f_{1} d f_{2} \\
\mathrm{HH}(t)=\int_{f_{1}=0.15^{+}}^{0.4} \int_{f_{2}=0.15^{+}}^{0.4} \mathrm{~B}\left(f_{1}, f_{2}, t\right) d f_{1} d f_{2}
\end{array}
$$




\subsection{Instantaneous Measures of Complexity}

\subsubsection{Instantaneous Lyapunov Exponents}

By expanding the quadratic regression described in Eq. 2 to the cubic order, and using a Fast Orthogonal Search algorithm, it is possible to estimate the complete Lyapunov Exponents (LE) spectrum at each moment in time [36], which provided additional and novel information about the dynamics of complexity and its variability. In this study, we use the Instantaneous Dominant Lyapunov Exponent (IDLE, $\lambda_{1}$ ), which is the first exponent of the LE spectrum $\lambda_{i}$ :

$$
\lambda_{i}=\frac{1}{\Delta N} \sum_{j=0}^{N-1} \ln R_{(j) i i}
$$

where $\Delta$ is the sampling time step, $N$ the data samples, and $R_{(j) i i}$ is part of the QR decomposition of the Jacobian of the time series [36].

\subsubsection{Instantaneous Entropy Measures}

In this study, the estimated instantaneous entropy measures refer to the inhomogeneous point-process approximate and sample entropy, $A_{\mathrm{I}}$ and $S_{\mathrm{I}}$, respectively [38]. These measures have their foundation in the instantaneous phase space estimation, in which the distance between two points is calculated through Kolmogorov-Smirnov (KS) distance (i.e. the maximum value of the absolute difference between two cumulative distribution functions) between the two pdfs associated to these two points. The time-varying radius $r(t)$ is instantaneously expressed as $r(t)=0.2 \sigma_{\mu_{\mathrm{e}(t)}}[38]$.

Of note, this instantaneous assessment of complexity opens the possibility of analyzing the proposed measures also in terms of variability of their evolution along time - an idea which we refer to as our complexity variability framework [38]. Moreover, we have shown that the dynamical estimates $A_{\mathrm{I}}$ and $S_{\mathrm{I}}$ are not substantially affected by the type of noise underlying the complex system, thus ensuring truly instantaneous tracking of the complexity of the underlying dynamical system [38]. 


\subsubsection{Feature vectors and statistical analysis}

All features were calculated instantaneously with a $\Delta=5 \mathrm{~ms}$ temporal resolution. In order to build subject-specific feature vectors, for every subject and for evey feature $X$, we condensed the information about the time-varying dynamics of $X$ through its median $\tilde{X}$ and its respective absolute deviation $\tilde{\tilde{X}}$ across time. As an exploratory/preliminary step, for each feature, we evaluated between-group differences in $\tilde{X}$ and $\tilde{X}$ for every feature using bivariate non parametric statistics (Mann-Whitney test) under the null hypothesis that the between-subject medians of the two groups are equal. Also, in order to investigate whether our instantaneous HRV estimates can provide additional within-disease information, we analysed univariate associations between these estimates and a battery of clinical/neuropsychological tests within out PD sample (Soearman Rank correlation, see section 2.2. for details).

\subsection{Single-subject classification and feature selection}

In order to investigate weather using heartbeat dynamics alone is able to aid in single-subject discrimination, we employed an automatic classification algorithm. Specifically, we chose the well-known SVM-based method of classification [34] due to its increasingly widespread use in biomedical literature. In this context, data gathered from each subject constitutes one multidimensional point in the feature space along with its label (healthy control/PD), and each feature constitutes a single dimension of this feature space. A multidimensional point was considered an outlier if z-scores associated to its dimensions were greater than 3. To assess the out-of-sample predictive accuracy of the system, we adopted a Leave-One-Out (LOO) procedure based on a Support Vector Machine (SVM)-based classifier [34]. Specifically, we employed a nu-SVM $(\mathrm{nu}=0.5)$ with a radial basis kernel function with

$\gamma=n^{-1}$, where $\mathrm{n}=22$ is equal to the number of features (see Table 1 for a complete list). Within the LOO scheme, the training set was normalized by subtracting the median value and dividing by the MAD over each dimension. These values were then used to normalize the example belonging to the test set. During the LOO procedure, this normalization step was 
performed on each fold. Classification results are summarized as balanced recognition accuracy (i.e. average of sensitivity and specificity) [49]. Additionally, in order to explore the relative importance of all features in the classification problem, we employed a support vector machine recursive feature elimination (SVM-RFE) procedure in a wrapper approach (RFE was performed on the training set of each fold and we computed the median rank for each feature over all folds). We specifically chose a recently developed nonlinear SVM-RFE which employes a radial basis function kernel and includes a correlation bias reduction strategy into the feature elimination procedure [41]. All analyses were performed using Matlab(C) (MathWorks, Natick, Massachusetts, USA) v8.4 and an additional toolbox for pattern recognition (LIBSVM [7]).

\section{Results}

In this study, point-process analyses yielded optimal NARL orders of $p=4$ and $q=2$. In 45 out of a total of 59 recordings, both KS plots and more than $98 \%$ of the autocorrelation samples fell within $95 \%$ confidence intervals. Of note, KS distances were as low as $0.0391 \pm 0.0060$. Instantaneous series from a representative PD patient and healthy control subject are shown in Fig. 3. Through our previously developed R-R interval error detection and correction algorithm [9], we corrected a total of 55 beats $(0.0718 \%)$ over all records. 

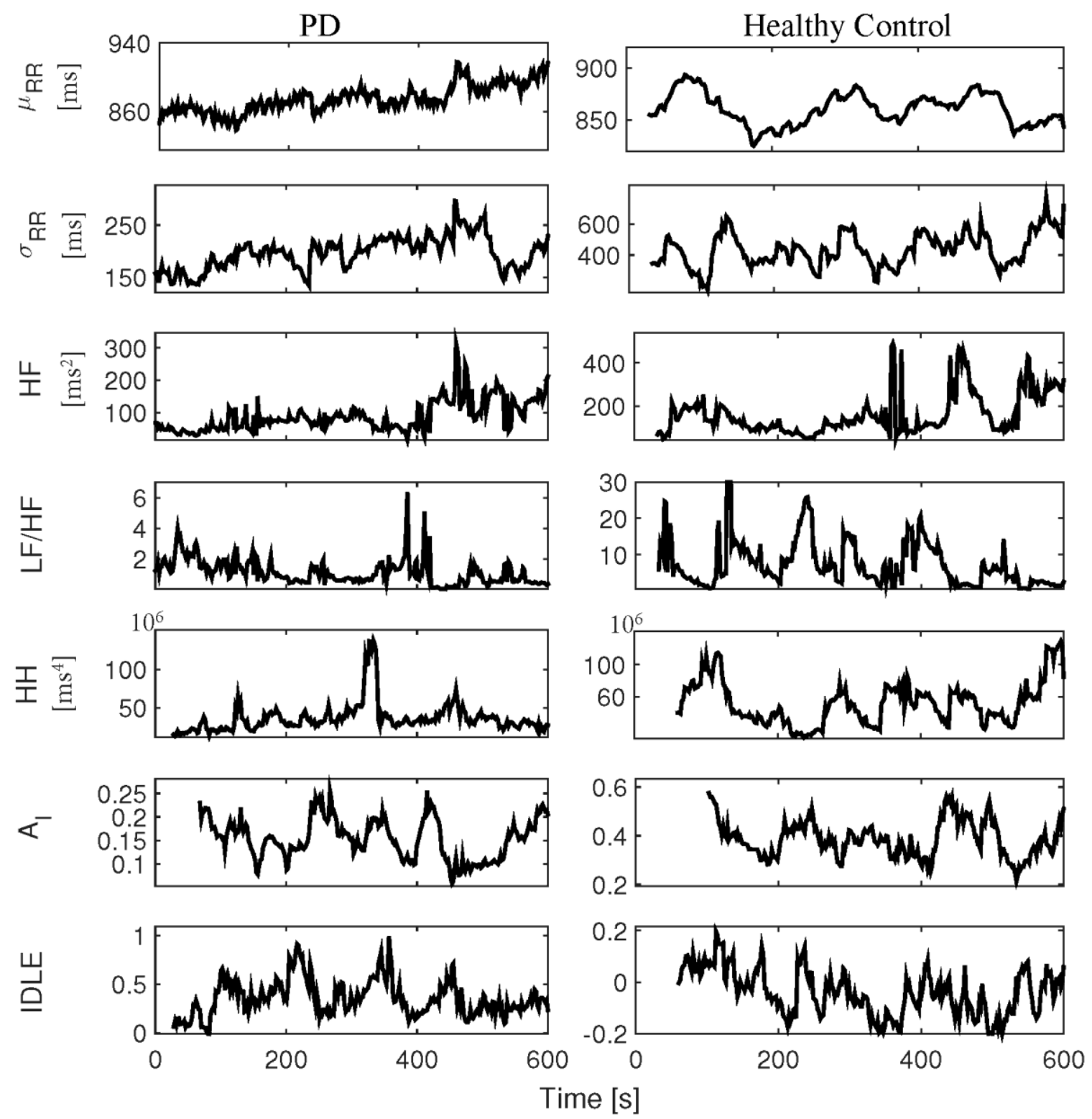

Figure 3: Instantaneous heartbeat estimates computed from a representative PD patient (left column) and a healthy control (right column), obtained through a point-process NARL model. From the top panel, the estimated mean $\mu_{\mathrm{RR}}(t)$, the standard deviation $\sigma_{\mathrm{RR}}(t)$, the high frequency (HF), the sympatho-vagal balance (LF/HF), the $\mathrm{HH}$ bispectral statistics, the inhomogeneous point-process approximate entropy $A_{I}$, and the instantaneous dominant Lyapunov exponent (IDLE) are depicted.

\subsection{Group differences in HRV features}

The results of group-wise univariate statistics in all features are shown in Table 1, and representative group-wise feature values for standard HRV features, higher order statistics (HOS) and complexity-related features are depicted in Fig. 4. 


\begin{tabular}{|c|c|c|c|c|}
\hline & Index & Parkinson's & Healthy & p-val \\
\hline \multirow{10}{*}{ Standard } & $\widetilde{\mu_{R R}}[\mathrm{~ms}]$ & $915.5 \pm 71.9$ & $918.3 \pm 101.2$ & 0.978 \\
\hline & $\widetilde{\widetilde{\mu_{R R}}}[\mathrm{~ms}]$ & $15.7 \pm 3.1$ & $15.7 \pm 3.7$ & 0.416 \\
\hline & $\widetilde{\sigma_{R R}}[\mathrm{~ms}]$ & $203.64 \pm 104.84$ & $272.46 \pm 117.84$ & 0.978 \\
\hline & $\widetilde{\widetilde{\sigma_{R R}}}[\mathrm{~ms}]$ & $55.40 \pm 23.63$ & $58.87 \pm 23.11$ & 0.725 \\
\hline & $L F\left[m s^{2}\right]$ & $184.2 \pm 119.13$ & $176.27 \pm 108.93$ & 0.807 \\
\hline & $\widetilde{L F}\left[m s^{2}\right]$ & $106.99 \pm 73.94$ & $76.79 \pm 36.86$ & 0.588 \\
\hline & $\overparen{H F}\left[m s^{2}\right]$ & $121.64 \pm 50.50$ & $141.44 \pm 78.20$ & 0.787 \\
\hline & $\widetilde{H F}\left[m s^{2}\right]$ & $36.81 \pm 17.58$ & $45.17 \pm 20.87$ & 0.551 \\
\hline & $L F / H F$ & $1.273 \pm 0.805$ & $1.262 \pm 0.608$ & 0.685 \\
\hline & $L \widetilde{F / H F}$ & $0.724 \pm 0.415$ & $0.547 \pm 0.229$ & 0.745 \\
\hline \multirow{6}{*}{ HOS } & $\widetilde{L L}\left[m s^{4}\right]$ & $530.54 \pm 348.34\left(10^{6}\right)$ & $815.34 \pm 622.93\left(10^{6}\right)$ & 0.070 \\
\hline & $\widetilde{L L}\left[m s^{4}\right]$ & $213.53 \pm 152.16\left(10^{6}\right)$ & $425.71 \pm 340.86\left(10^{6}\right)$ & 0.099 \\
\hline & $\widetilde{L H}\left[m s^{4}\right]$ & $1839.7 \pm 1018.1\left(10^{6}\right)$ & $2638.7 \pm 1224.4\left(10^{6}\right)$ & 0.807 \\
\hline & $\widetilde{L H}\left[m s^{4}\right]$ & $745.14 \pm 267.69\left(10^{6}\right)$ & $751.26 \pm 426.96\left(10^{6}\right)$ & 0.978 \\
\hline & $\widehat{H H}\left[m s^{4}\right]$ & $9348.4 \pm 5634.4\left(10^{6}\right)$ & $7953.1 \pm 4155.3\left(10^{6}\right)$ & 0.203 \\
\hline & $\widetilde{H H}\left[m s^{4}\right]$ & $3892.6 \pm 2604.5\left(10^{6}\right)$ & $3663.2 \pm 1927.4\left(10^{6}\right)$ & 0.244 \\
\hline \multirow{6}{*}{ Complexity } & $I \widehat{D L E}$ & $-0.004 \pm 0.027$ & $-0.034 \pm 0.035$ & 0.066 \\
\hline & $I \widetilde{D L E}$ & $0.0796 \pm 0.0140$ & $0.0596 \pm 0.0136$ & 0.032 \\
\hline & $\widetilde{A_{I}}$ & $0.2940 \pm 0.083$ & $0.2943 \pm 0.060$ & 0.473 \\
\hline & $\widetilde{A_{I}}$ & $0.0444 \pm 0.0069$ & $0.0553 \pm 0.0141$ & 0.110 \\
\hline & $\widehat{S_{I}}$ & $0.2388 \pm 0.1068$ & $0.3039 \pm 0.0696$ & 0.350 \\
\hline & $\widetilde{S_{I}}$ & $0.0565 \pm 0.0114$ & $0.0668 \pm 0.0171$ & 0.516 \\
\hline
\end{tabular}

Table 1: Statistical analysis of group differences in all condensed features between PD and healthy controls. HOS= Higher Order Statistics. For a feature $X$, intervals are expressed as $X=$ Median $(X) \pm M A D(X)$. Bold indicates a statistically significant difference.

No group-wise significant differences were found in higher order features except for $\widetilde{\widetilde{I D L E}}$, i.e., one of the heartbeat complexity variability indices. Classical HRV indices were not significantly different between groups. 

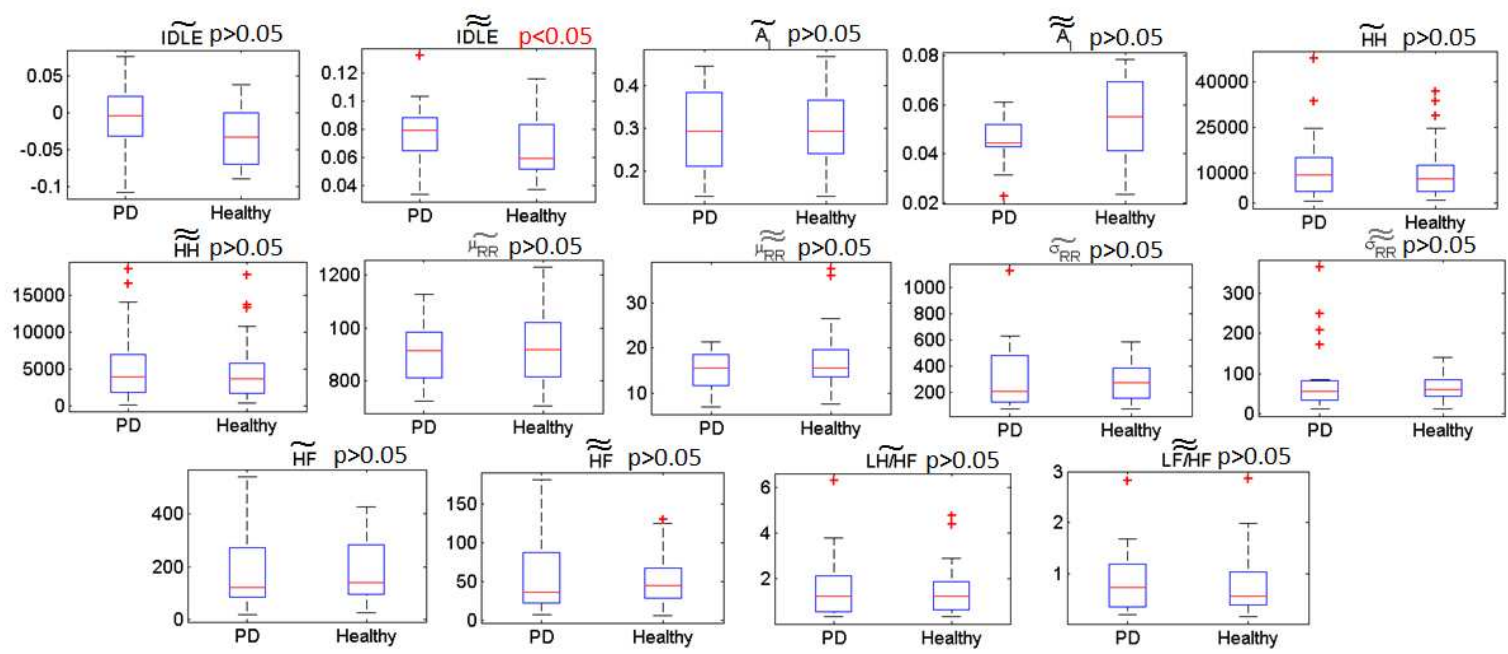

Figure 4: Box-plot plots of representative heartbeat features estimated through inhomogeneous point-process NARL modeling. Red asterisks are outliers. P-values are gathered from Mann-Whitney non-parametric tests.

\subsection{Association between HRV features and clinical/neuropsychological scores}

The results of exploring univariate associations between our feature vectors and clinical/motor, affective, and neuropsychological scores are shown in Tables 2, 3, and 4. In particular, Table 2 reports significant pairwise non-parametric Spearman correlation beween our HRV estimates and clinical/motor patient evaluations (UPDRSII/III), Table 3 reports significant pairwise non-parametric Spearman correlation beween our HRV estimates and affective state (GDS), and Table 4 reports the significant pairwise non-parametric Spearman correlation beween neuropsychological patient evaluations and HRV estimates.

\begin{tabular}{|c|c|c|c|}
\hline Clinical/Motor Scales & \multirow{2}{*}{ Index } & Test & $\rho$ \\
\hline \multirow{2}{*}{ Standard } & $\widetilde{\widetilde{\mu_{R R}}}$ & UPDRS II & -0.558 \\
\cline { 3 - 4 } & $\widetilde{U}$ & UPDRS III & -0.518 \\
\hline \multirow{2}{*}{ HOS } & $\widetilde{L L}$ & UPDRS II & -0.656 \\
\cline { 3 - 4 } & $\widetilde{\widetilde{L L}}$ & UPRS III & -0.641 \\
\cline { 3 - 4 } & & UPDRS II & -0.583 \\
\hline & & &
\end{tabular}

Table 2: Significant correlations (non-parametric Spearman Rank correlation $\rho$ with $p<0.05$ ) between HRV estimates and clinical/motor scores from Unified Parkinson's Disease Rating Scale (UPDRS). All other associations did not reach statistical significance. 


\begin{tabular}{|c|c|c|c|}
\hline Affective Scales & Index & Test & $\rho$ \\
\hline \multirow{2}{*}{ Standard } & $\widetilde{\mu_{R R}}$ & GDS & 0.499 \\
\hline \multirow{2}{*}{ HOS } & $\widetilde{L L}$ & GDS & 0.501 \\
\cline { 2 - 4 } & $\widetilde{\widetilde{L L}}$ & GDS & 0.501 \\
\hline
\end{tabular}

Table 3: Significant correlations (non-parametric Spearman Rank correlation $\rho$ with $p<0.05$ ) between HRV estimates and affective scores from Geriatric Depression Scale (GDS). All other associations did not reach statistical significance.

\begin{tabular}{|c|c|c|c|}
\hline Neuropsychology & Index & Test & $\rho$ \\
\hline \multirow{15}{*}{ Standard } & \multirow{4}{*}{$\widetilde{\sigma_{R R}}$} & TMT B & 0.560 \\
\hline & & FAB & -0.513 \\
\hline & & Boston_60 & -0.570 \\
\hline & & Benton_JLO & -0.581 \\
\hline & $\widetilde{\sigma_{R R}}$ & Boston_60 & -0.603 \\
\hline & \multirow{2}{*}{$\widetilde{L F}$} & Boston_60 & -0.556 \\
\hline & & Benton_JLO & -0.623 \\
\hline & \multirow{2}{*}{$\widetilde{\widetilde{L F}}$} & Boston_60 & -0.619 \\
\hline & & Benton_JLO & -0.595 \\
\hline & \multirow{2}{*}{$H F$} & Boston_60 & -0.682 \\
\hline & & Benton_JLO & -0.671 \\
\hline & \multirow{2}{*}{$\widetilde{\widehat{H F}}$} & Boston_60 & -0.634 \\
\hline & & Benton_JLO & -0.622 \\
\hline & $L F / H F$ & Analogie_W & -0.658 \\
\hline & $L \widetilde{F / H F}$ & Stroop_E & -0.503 \\
\hline \multirow{8}{*}{ HOS } & $\widetilde{L L}$ & Benton_JLO & -0.677 \\
\hline & $\widetilde{\widetilde{L L}}$ & Benton_JLO & -0.594 \\
\hline & $L H$ & Benton_JLO & -0.511 \\
\hline & \multirow[b]{2}{*}{$\widetilde{L H}$} & Boston_60 & -0.499 \\
\hline & & Benton_JLO & -0.508 \\
\hline & $\overparen{H H}$ & Boston_60 & -0.566 \\
\hline & $\widehat{H H}$ & Boston_60 & -0.514 \\
\hline & $H H$ & Benton_JLO & -0.526 \\
\hline \multirow{3}{*}{ Complexity } & $I \widehat{D L E}$ & Analogie_W & 0.500 \\
\hline & $I \widetilde{D L E}$ & Stroop_E & 0.680 \\
\hline & $\widetilde{A_{I}}$ & TMT_B & 0.582 \\
\hline
\end{tabular}

Table 4: Significant correlations (non-parametric Spearman Rank correlation $\rho$ with $p<0.05$ ) between HRV estimates and neuropsychological scores from Trail Making Test (TMT_B), Frontal Assessment Battery (FAB), Boston Naming Test (Boston_60), Benton Judgment of Line Orientation (Benton_JLO), Wechsler Adult Intelligence Scale Analogy Subtest (Analogie_W), Stroop Test (Stroop_E). All other did not reach statistical significance. 


\subsection{Single-subject classification and feature selection}

Classification results are reported in terms of balanced recognition accuracy (see paragraph 2.6 for definition) as well as by reporting the confusion matrix [22]. The generic element $r_{i j}$ of the confusion matrix indicates the percentage of instances belonging to class $i$ classified as belonging to class $j$ - a "more diagonal" confusion matrix therefore corresponds to a better classification. Table 5 shows the confusion matrix as well as the balanced accuracy in discerning patients with PD from healthy subjects when taking into account all 22 features. In this case, the balanced accuracy was $73.47 \%$.

\begin{tabular}{|c|c|c|}
\hline & Healthy controls & PD Patients \\
\hline \hline Healthy controls & 70.83 & 29.17 \\
\hline PD Patients & 24.00 & 76.00 \\
\hline
\end{tabular}

Balanced Accuracy: 73.47; Specificity:70.83; Sensitivity: 76.

Table 5: Confusion matrix of SVM classifier (PD vs. Healthy Controls) using all features. Values are expressed as percentages.

Further, the complete list of features ordered by their median rank over every fold computed during the LOO procedure is reported in Table 6.

\begin{tabular}{|c|c|}
\hline Rank & Feature \\
\hline 1 & $\widetilde{\widetilde{A_{I}}}$ \\
\hline 2 & $\widetilde{L H}$ \\
\hline 3 & $\widetilde{\widetilde{L L}}$ \\
\hline 4 & $\widetilde{\widetilde{L F}}$ \\
\hline 5 & $\widetilde{\widetilde{D L E}}$ \\
\hline 6 & $\widetilde{S_{I}}$ \\
\hline 7 & $\widetilde{H_{F}}$ \\
\hline 8 & $\widetilde{I D L E}$ \\
\hline 9 & $\widetilde{A_{I}}$ \\
\hline 10 & $\widetilde{\mu_{R R}}$ \\
\hline 11 & $\widetilde{\widetilde{S_{I}}}$ \\
\hline
\end{tabular}

\begin{tabular}{|c|c|}
\hline Rank & Feature \\
\hline 12 & $\widetilde{\sigma_{R R}}$ \\
\hline 13 & $\widetilde{\widetilde{\sigma_{R R}}}$ \\
\hline 14 & $\widetilde{L L}$ \\
\hline 15 & $\widetilde{H F}$ \\
\hline 16 & $\widetilde{L F}$ \\
\hline 17 & $\widetilde{\widetilde{L H}}$ \\
\hline 18 & $L \widetilde{F / H} F$ \\
\hline 19 & $\widetilde{\mu_{R R}}$ \\
\hline 20 & $\widetilde{\widetilde{H H}}$ \\
\hline 21 & $\widetilde{\widetilde{F H}} F$ \\
\hline 22 & $\widetilde{H H}$ \\
\hline
\end{tabular}

Table 6: Features ordered by median rank across folds when performing feature selection through the SVM-RFE 
procedure.

In addition to the above analyses, we repeated the classification analysis employing only features related to the central tendency (median) of all features, hence excluding measures of variability. Results of this classification are shown in Table 7. In this case, the balanced accuracy was $61.91 \%$.

\begin{tabular}{|c|c|c|}
\hline & Healthy controls & PD Patients \\
\hline \hline Healthy controls & 47.83 & 52.17 \\
\hline PD Patients & 24.00 & 76.00 \\
\hline
\end{tabular}

Balanced Accuracy: 61.91; Specificity:47.83; Sensitivity: 76.

Table 7: Confusion matrix of SVM classifier (PD vs. Healthy Controls) excluding features related to instantaneous variability. Values are expressed as percentages. 


\section{Discussion and Conclusion}

In this study we examined instantaneous linear, nonlinear, and complex cardiovascular dynamics in PD, investigating both differences with a healthy control group and within-disease relationships between our features and neuropsychological/clinical scores. We demonstrate that the information most useful to discriminate between PD patients from controls is derived from higher-order, instantaneus statistics of heartbeat dynamics as well as from their variability over time. Additionally, we show that both linear and nonlinear model features significantly correlate with clinical/motor, affective, and neuropsychological assesments of our patient population, pointing towards an added value of such measures in stratifying disease subtypes as well as disease-related disabilities in the motor as well as cognitive domain. To our knowledge, this is the first study which investigates and develops single-subject characterization and classification in PD based on heartbeat dynamics alone. Our patient population was comprised of 15 de novo PD patients and 15 patients with more advanced PD, which necessarily were on dopaminergic drugs. We feel that this balance is the best strategy in guaranteeing sufficient variability in disease-related motor and cognitive alterations while mitigating the risk of confounding classification results.

We employed recently defined instantaneous estimates of heartbeat complex dynamics such as the dominant Lyapunov exponent and instantaneous approximate and sample entropy, as well as instantaneous nonlinear bispectral measures and linear estimates defined in the time and frequency domain. The modeling technique underlying these estimates is based on the theory of inhomogeneous point processes, whose $p d f$ is parametrized through a nonlinear autoregressive model with Laguerre expansion of the Wiener-Volterra terms. These terms are expanded up to the cubic order, and ensure optimal and parsimonious estimation of the intrinsic dynamical and stochastic structure of heartbeat dynamics. Of note, measures of instantaneous complexity are not affected by the statistical properties of the physiological noise behind the observed dynamics [36,38], thus ensuring that when performing group comparisons like the one presented in this study, differences are associated with the actual underlying dynamics as opposed to possible alterations in the noise statistics (e.g., a transition 
from $1 / f$ noise to Gaussian noise). We employed these real-time estimates under the hypothesis that they would aid in improving the current knowledge of cardiovascular disfunction in PD as well as with the aim of building a simple (i.e., based only on cardiovascular variability) decision support framework able to discern a PD patients from healthy controls.

In PD, autonomic system disturbances (a.k.a. dysautonomia) reflect neurodegenerative processes which are known to reach beyond the nigrostriatal dopaminergic system $[44,45]$, and follow a largely independent pathological progression when compared to dopaminergic symptoms. As a consequence, dysautonomia can appear in all stages of PD. Additionally, it has been shown that the assessment of cardiovascular autonomic failure can aid in early recognition and treatment of PD $[2,18]$, and previous studies have demonstrated ANS-related differences among PD and parkinsonisms such as Multiple System Atrophy (MSA) and Progressive supranuclear palsy (PSP) $[46,47]$.

Also, while dysautonomic symptoms may have a significant impact on daily activities as well as quality of life [11], the evaluation of ANS dysfunctions relies on a large battery of autonomic tests only accessible in highly specialized laboratories $[46,47,48]$ and it is currently associated with a large amount of diagnostic and financial overhead. A better understanding, early recognition and treatment of ANS failure in PD may a) have a significant impact on daily activities and quality of life and b) aid in the differential diagnosis of Parkinsonisms, or even in the characterization of cognitive profile of PD patients - to this end, novel systems able to support the diagnosis of cardiovascular dysautonomia are highly desirable.

To the best of our knowledge, prior studies have focused on group comparisons, ofter reaching contradictory results $[1,3,16,20,21,25,28,29,32,33,35]$. It should be noted that, even if a significant statistical difference associated to a certain feature is consistently revealed across several studies, this is not sufficient to demonstrate its potential application to the clinical necessity of single-subject characterization. This motivated us to reach beyond conventional group comparisons and explore a classification algorithm which a) could discern between PD and healthy controls and b) has the intrinsic capability of being generalized to novel, unseen (to the algorithm) data. 
As a preliminary, exploratory step, we performed a statistical analysis between PD and healthy controls (see Table 1) which confirmed our hypothesis that inter-subject variability would blur most group differences in cardiovascular dynamics except for higher order complexity measures derived from the time domain, such as $\overline{\overline{I D L E}}$. This result is consistent with our previous findings describing how differences in autonomic complexity associated with pathological states are best detected by examining the variability of a time-varying complexity measure (as opposed to standard estimates of complexity), and that higher complexity variability is consistently associated with pathological states [36]. In this context, the results of this paper further corroborate the use of real-time estimates of heartbeat dynamics through point-process modeling to obtain additional information about ANS complexity and its modulations in disease. While considering the group comparisons in all features as separate, group differences in instantaneous complexity would not remain significant after correction for multiple comparisons, group trends associated to $\overline{\overline{I D L E}}$ and other instantaneous features provide meaningful insights on the patho-physiology of cardiovascular dynamics in PD. Given the subtle nature of the alterations under investigation and the highly nonlinear structure of our features, a larger study would be necessary to test a high number of simultaneous hypotheses about multiple HRV features.

In order to explore the associations between our instantaneous HRV estimates, and within-disease mechanisms of motor as well as cognitive impairments, we performed a non-parametric correlation analysis between all features and clinical/neuropsychological scores (see Tables 2-4). Visuospatial ability as evaluated by the Benton Judgment of Line Orientation, was found to be negatively correlated with all spectral and bispectral measures as well as their variability, possibily indicating a relationship between increased parasympathetic activity and visuospatial skills in PD. The same measures (except for $\widetilde{L L}$, and $\widetilde{\widetilde{L L}}$ ) correlated negatively also with language ability as evaluated by the Boston naming test. Motor impairment, as evaluated by UPDRS II/III, correlated negatively with $\widetilde{L L}$ and $\widetilde{\widetilde{L L}}$, as well as $\widetilde{\widetilde{\mu_{R}}}$, possibly indicating an independent relationship of these features with motor as opposed to cognitive ability in PD Accordingly, $\widetilde{L L}$ and $\widetilde{\widetilde{L L}}$ also correlated positively with GDS. $\widetilde{\widetilde{I D L E}}$ was positively correlated 
with the Stroop (E) score, indicating an association between complexity variability and information processing. The results of the trail making test B were positively associated with both $\widetilde{A_{I}}$ and $\widetilde{\sigma_{R R}}$, the former indicating a relationship between heartbeat complexity and disease-related attention deficit, in possible agreement with our previous findings of an increased instability of heartbeat dynamics in PD [4].

The automatic classification was performed using nu-SVM, and included a LOO procedure. Results showed a balanced accuracy of $73.47 \%$ when all features (central tendency and time-variability) were taken into account (see Table 5). This accuracy results from the combination of a specificity of $70.83 \%$, and a sensitivity of $76 \%$. Given that, to the best of our knowledge, our result is the first attempt to demonstrate that the discrimination of patients suffering from parkinsonisms by ANS characterization can be supported by analysis based on heartbeat dynamics alone, we consider this balanced classification accuracy as satisfactory. While the superior effectiveness of our instantaneous heartbeat linear and nonlinear estimates in discriminating physio-pathological states, also as compared with traditional not-in-real-time estimates such as LF and HF power estimated through FFT or autoregressive models, has already been demonstrated in our previous methodological papers $[5,36,37]$, in this study we specifically investigated the added value of variability-related features in classifying and characterizing PD patients. To this end, we repeated the classification analysis (PD vs. heathy controls) including only features related to the central tendencies of all featues (see Table 7). In this case, the balanced accuracy of classification dropped to $61.91 \%$, with a sensitivity of $76 \%$ and specificity of $47.83 \%$. Furthermore, a rigorous SVM-RFE procedure revealed that the most informative features are derived from the instantaneous variability of our instantaneous estimates (see Table 6). Of note, the SVM-RFE implemented in this study uses a radial basis function kernel, and reduces bias due to highly correlated features [41].

In summary, we demonstrate that the use of instantaneous heartbeat dynamics model, yields linear, nonlinear, and complex statistics in a time-resolved manner, and allows to both significantly improve balanced classification accuracy when comparing PD to controls. Given that the proposed methodology only requires an ECG monitoring system which, nowadays, is 
widely available thanks to the use of more portable devices (e.g., standard ECG holter), it would be possible to obtain this information without a full neurological examination. In the case of Parkinsonisms, this would allow for a more rapid and efficient initial patient characterization. Also, given that several of our novel higher order features significantly correlate with motor and cognitive scores, the proposed framework could conceivably be useful in providing additional information for elucidating within-disease mechanisms of ANS-related progression of disability and cognitive impairment. This would aid the longitudinal monitoring of disease progression (after appropriate validation through longitudinal HRV studies in PD) through incorporation of a routine monitoring of ANS function in periodical patient evaluation. Finally, concerning the therapeutic management of PD, after proper characterization of the effects of dopaminergic therapy on our instantaneous HRV measures, the latter could also provide an additional monitoring instrument for drug related side-effects associated with autonomic functioning.

Future work will investigate a parameter optimization procedure for SVM classifiers, as well as will focus on a rigorous comparison with other automatic classification methods, to be benchmarked within a larger sample size.

\section{Acknowledgements}

The research leading to these results has received partial funding from the Department of Anesthesia, Critical Care \& Pain Medicine, Massachusetts General Hospital, and Harvard Medical School, Boston, MA, USA, and European Union Seventh Framework Programme FP7/2007-2013 under Grant No. 601165 of the project "WEARHAP". 


\section{References}

[1] Appenzeller, O., Goss, J.E.: Autonomic deficits in parkinson's syndrome. Archives of neurology 24(1), 50-57 (1971)

[2] Asahina, M., Vichayanrat, E., Low, D.A., lodice, V., Mathias, C.J.: Autonomic dysfunction in parkinsonian disorders: assessment and pathophysiology. Journal of Neurology, Neurosurgery \& Psychiatry 84(6), 674-680 (2013)

[3] Awerbuch, G.I., Sandyk, R.: Autonomic functions in the early stages of parkinson's disease. International journal of neuroscience 64(1-4), 7-14 (1992)

[4] Barbieri, R., Citi, L., Valenza, G., Guerrisi, M., Orsolini, S., Tessa, C., Diciotti, S., Toschi, N.: Increased instability of heartbeat dynamics in parkinson's disease. In: Computing in Cardiology Conference (CinC), 2013, pp. 89-92. IEEE (2013)

[5] Barbieri, R., Matten, E., Alabi, A., Brown, E.: A point-process model of human heartbeat intervals: new definitions of heart rate and heart rate variability. American Journal of Physiology-Heart and Circulatory Physiology 288(1), H424 (2005)

[6] Barbieri, R., Valenza, G., Citi, L., Guerrisi, M., Orsolini, S., Tessa, C., Diciotti, S., Toschi, N.: Lower instantaneous entropy of heartbeat dynamics characterizes cognitive impairment in parkinson's disease. In: Computing in Cardiology Conference (CinC), 2014, pp. 81-84. IEEE (2014)

[7] Chang, C.C., Lin, C.J.: LIBSVM: A library for support vector machines. ACM Transactions on Intelligent Systems and Technology 2, 27:1-27:27 (2011). Software available at http://www.csie.ntu.edu.tw/ cjlin/libsvm

[8] Chang, H.A., Chang, C.C., Kuo, T.B., Huang, S.Y.: Distinguishing bipolar ii depression from unipolar major depressive disorder: Differences in heart rate variability. The World Journal of Biological Psychiatry (0), 1-10 (2015)

[9] Citi, L., Brown, E.N., Barbieri, R.: A real-time automated point-process method for the detection and correction of erroneous and ectopic heartbeats. Biomedical Engineering, IEEE Transactions on 59(10), 2828-2837 (2012)

[10] Costa, M.D., Goldberger, A.L.: Generalized multiscale entropy analysis: Application to quantifying the complex volatility of human heartbeat time series. Entropy 17(3), 1197-1203 (2015)

[11] Fanciulli, A., Strano, S., Colosimo, C., Caltagirone, C., Spalletta, G., Pontieri, F.: The potential prognostic role of cardiovascular autonomic failure in $\alpha$-synucleinopathies. European 
Journal of Neurology 20(2), 231-235 (2013)

[12] Forno, L.S.: Neuropathology of parkinson's disease. Journal of Neuropathology \& Experimental Neurology 55(3), 259-272 (1996)

[13] Gibb, W., Lees, A.: The relevance of the lewy body to the pathogenesis of idiopathic parkinson's disease. Journal of Neurology, Neurosurgery \& Psychiatry 51(6), 745-752 (1988)

[14] Glass, L.: Dynamical disease: Challenges for nonlinear dynamics and medicine. Chaos: An Interdisciplinary Journal of Nonlinear Science 25(9), 097,603 (2015)

[15] Granger, C., Joyeux, R.: An introduction to long-memory time series models and fractional differencing. Journal of time series analysis 1(1), 15-29 (1980)

[16] Haapaniemi, T., Pursiainen, V., Korpelainen, J., Huikuri, H., Sotaniemi, K., Myllylä, V.: Ambulatory ecg and analysis of heart rate variability in parkinson's disease. Journal of neurology, neurosurgery \& psychiatry 70(3), 305-310 (2001)

[17] Hoehn, M.M., Yahr, M.D.: Parkinsonism: onset, progression, and mortality. Neurology 50(2), 318-318 (1998)

[18] Jain, S., Goldstein, D.S.: Cardiovascular dysautonomia in parkinson disease: from pathophysiology to pathogenesis. Neurobiology of disease 46(3), 572-580 (2012)

[19] Jankovic, J., McDermott, M., Carter, J., Gauthier, S., Goetz, C., Golbe, L., Huber, S., Koller, W., Olanow, C., Shoulson, I., et al.: Variable expression of parkinson's disease a base-line analysis of the dat atop cohort. Neurology 40(10), 1529-1529 (1990)

[20] Kallio, M., Suominen, K., Bianchi, A., Mäkikallio, T., Haapaniemi, T., Astafiev, S., Sotaniemi, K.A., Myllylä, V.V., Tolonen, U.: Comparison of heart rate variability analysis methods in patients with parkinson's disease. Medical and Biological Engineering and Computing 40(4), 408-414 (2002)

[21] Kiyono, K., Hayano, J., Kwak, S., Watanabe, E., Yamamoto, Y.: Non-gaussianity of low frequency heart rate variability and sympathetic activation: lack of increases in multiple system atrophy and parkinson disease. Frontiers in physiology 3 (2012) (1998)

[22] Kohavi, R., Provost, F.: Glossary of terms. Machine Learning 30(June), 271-274

[23] Litvan, I., Goldman, J.G., Tröster, A.I., Schmand, B.A., Weintraub, D., Petersen, R.C., Mollenhauer, B., Adler, C.H., Marder, K., Williams-Gray, C.H., et al.: Diagnostic criteria for mild cognitive impairment in parkinson's disease: Movement disorder society task force guidelines. Movement Disorders 27(3), 349-356 (2012) 
[24] Lo, M.T., Chang, Y.C., Lin, C., Young, H.W.V., Lin, Y.H., Ho, Y.L., Peng, C.K., Hu, K.: Outlier-resilient complexity analysis of heartbeat dynamics. Scientific reports $\mathbf{5}$ (2015)

[25] Ludin, S.M., Steiger, U.H., Ludin, H.: Autonomic disturbances and cardiovascular reflexes in idiopathic parkinson's disease. Journal of neurology 235(1), 10-15 (1987)

[26] Magerkurth, C., Schnitzer, R., Braune, P.D.D.S.: Symptoms of autonomic failure in parkinsonõs disease: prevalence and impact on daily life. Clinical Autonomic Research 15(2), 76-82 (2005)

[27] Marmarelis, V.: Identification of nonlinear biological system using laguerre expansions of kernels. Ann. Biomed. Eng. 21, 573-589 (1993)

[28] Meco, G., Pratesi, L., Bonifati, V.: Cardiovascular reflexes and autonomic dysfunction in parkinson's disease. Journal of neurology 238(4), 195-199 (1991)

[29] Netten, P.M., de Vos, K., Horstink, M.W., Hoefnagels, W.H.: Autonomic dysfunction in parkinson's disease, tested with a computerized method using a finapres device. Clinical Autonomic Research 5(2), 85-89 (1995)

[30] Orimo, S., Uchihara, T., Nakamura, A., Mori, F., Kakita, A., Wakabayashi, K., Takahashi, H.: Axonal $\alpha$-synuclein aggregates herald centripetal degeneration of cardiac sympathetic nerve in parkinson's disease. Brain 131(3), 642-650 (2008)

[31] on Rating Scales for Parkinson's Disease, M.D.S.T.F., et al.: The unified parkinson's disease rating scale (updrs): status and recommendations. Movement disorders: official journal of the Movement Disorder Society 18(7), 738 (2003)

[32] Porta, A., Castiglioni, P., Di Rienzo, M., Bari, V., Bassani, T., Marchi, A., Takahashi, A.C., Tobaldini, E., Montano, N., Catai, A.M., et al.: Short-term complexity indexes of heart period and systolic arterial pressure variabilities provide complementary information. Journal of Applied Physiology 113(12), 1810-1820 (2012)

[33] Sachs, C., Berglund, B., Kaijser, L.: Autonomic cardiovascular responses in parkinsonism: effect of levodopa with dopa-decarboxylase inhibition. Acta neurologica scandinavica 71(1), 37-42 (1985)

[34] Schölkopf, B., Smola, A.J., Williamson, R.C., Bartlett, P.L.: New support vector algorithms. Neural computation 12(5), 1207-1245 (2000)

[35] Senard, J., Rai, S., Lapeyre-Mestre, M., Brefel, C., Rascol, O., Rascol, A., Montastruc, J.: Prevalence of orthostatic hypotension in parkinsonõs disease. Journal of Neurology, Neurosurgery \& Psychiatry 63(5), 584-589 (1997) 
[36] Valenza, G., Citi, L., Barbieri, R.: Estimation of instantaneous complex dynamics through lyapunov exponents: a study on heartbeat dynamics. PloS one 9(8), e105,622 (2014)

[37] Valenza, G., Citi, L., Scilingo, E.P., Barbieri, R.: Point-process nonlinear models with laguerre and volterra expansions: Instantaneous assessment of heartbeat dynamics. Signal Processing, IEEE Transactions On 61(11), 2914-2926 (2013)

[38] Valenza, G., Citi, L., Scilingo, E.P., Barbieri, R.: Inhomogeneous point-process entropy: An instantaneous measure of complexity in discrete systems. Physical Review E 89(5), 052,803 (2014)

[39] Valenza, G., Nardelli, M., Bertschy, G., Lanata, A., Scilingo, E.: Mood states modulate complexity in heartbeat dynamics: A multiscale entropy analysis. EPL (Europhysics Letters) 107(1), 18,003 (2014)

[40] Westwick, D., Kearney, R.: Explicit least-squares methods, in Identification of nonlinear physiological systems, vol. 7. Wiley-IEEE Press (2003)

[41] Yan, K., Zhang, D.: Feature selection and analysis on correlated gas sensor data with recursive feature elimination. Sensors and Actuators B: Chemical, 212, 353-363 (2015)

[42] Acharya, U. R., Joseph, K. P., Kannathal, N., Lim, C. M., \& Suri, J. S.: Heart rate variability: a review. Medical and biological engineering and computing, 44(12), 1031-1051 (2006).

[43] Postuma, R. B., Aarsland, D., Barone, P., Burn, D. J., Hawkes, C. H., Oertel, W., \& Ziemssen, T. Identifying prodromal Parkinson's disease: Pre-Motor disorders in Parkinson's disease. Movement Disorders, 27(5), 617-626 (2012).

[44] Braak H, Ghebremedhin E, Rüb U, Bratzke H, Del Tredici K (2004): Stages in the development of Parkinson's disease-related pathology. Cell Tissue Res 318:121-134.

[45] Braak H, Del Tredici K (2008): Nervous system pathology in sporadic Parkinson disease. Neurology 70:1916-1925.

[46] Asahina, M., Vichayanrat, E., Low, D. A., Iodice, V., \& Mathias, C. J. (2013). Autonomic dysfunction in parkinsonian disorders: assessment and pathophysiology. Journal of Neurology, Neurosurgery \& Psychiatry, 84(6), 674-680.

[47] Kimpinski, K., Iodice, V., Burton, D. D., Camilleri, M., Mullan, B. P., Lipp, A., ... \& Low, P. A. (2012). The role of autonomic testing in the differentiation of Parkinson's disease from multiple system atrophy. Journal of the neurological sciences, 317(1), 92-96.

[48] Lipp, A., Sandroni, P., Ahlskog, J. E., Fealey, R. D., Kimpinski, K., Iodice, V., ... \& 
Low, P. A. (2009). Prospective differentiation of multiple system atrophy from Parkinson disease, with and without autonomic failure. Archives of neurology, 66(6), 742-750.

[49] Brodersen, K.H.; Ong, C.S.; Stephan, K.E.; Buhmann, J.M. (2010). "The balanced accuracy and its posterior distribution". Proceedings of the 20th International Conference on Pattern Recognition: 3121-24.

[50] Bonuccelli, U., C. Lucetti, P. Del Dotto, R. Ceravolo, G. Gambaccini, S. Bernardini, G. Rossi, and A. Piaggesi. (2003) "Orthostatic hypotension in de novo Parkinson disease." Archives of neurology 60, no. 10: 1400-1404.

[51] Litvan, I., Goldman, J. G., Tröster, A. I., Schmand, B. A., Weintraub, D., Petersen, R. C., ... \& Emre, M. (2012). Diagnostic criteria for mild cognitive impairment in Parkinson's disease: Movement Disorder Society Task Force guidelines. Movement Disorders, 27(3), 349-356. 